\title{
RESILIENCIA COMUNITARIA ANTE RIESGO DE TSUNAMI EN LA COSTA NEOLIBERAL: EL CASO DE LA COMUNA DE EL QUISCO, REGIÓN DE VALPARAÍSO, CHILE.
}

\section{COMMUNITY RESILIENCE TO TSUNAMI RISK ON THE NEOLIBERAL COAST: THE CASE OF THE QUISCO COMMUNE, VALPARAISO REGION, CHILE.}

\section{Simón Inzunza General*}

RESUMEN: Ante el aumento del riesgo de desastre ante tsunami en la zona costera, se hace necesario el desarrollo de lineamientos que aborden esta problemática y, en esta línea, el paradigma de la resiliencia comunitaria ha tomado preponderancia para su generación. Se evaluó la resiliencia comunitaria ante tsunami en la comuna de El Quisco, Chile, y se estableció cómo el urbanismo neoliberal, específicamente la turistización del territorio, afecta esta dimensión. Se aplicó encuestas en tres unidades de análisis, resultando dos factores de resiliencia comunitaria ante tsunami a través de un análisis de componentes principales: "conocimiento del lugar, redes y preparación ante tsunami" y "cohesión y percepción de la amenaza". Asimismo, se indagó sobre vulnerabilidad social, años de residencia, morfología y tipología urbana, elementos que influyen en la resiliencia y se contextualizan por la gestión neoliberal del territorio. U 1 resultó en zona turística y alcanzó el valor de resiliencia más bajo; U2 es zona turística-residencial y obtuvo una cifra de resiliencia intermedia; U3 es zona residencial y alcanzó nivel de resiliencia mayor. Se establece que en aquellas áreas másturistizadas, las relaciones sociales están en mayor medida atomizadas y hay menos relación con el entorno próximo, disminuyendo consecuentemente la resiliencia comunitaria ante tsunami. Se concluye que, si bien es importante relevar el rol agentivo de las comunidades frente a la reducción del riesgo, las causas de los desastres responden a factores estructurales que escapan del campo de acción de la acción comunitaria, por lo que las acciones deben ir, en primer lugar, en línea a la reducción de la vulnerabilidad y la exposición.

Palabras clave: gestión del riesgo, tsunami, resiliencia comunitaria, turistización del territorio, urbanismo neoliberal. 


\begin{abstract}
Given the increased risk of tsunami disaster in the coastal zone, it is necessary to develop guidelines to address this problem and, in this line, the paradigm of community resilience has taken preponderance for its generation. Community resilience to tsunami in the commune of El Quisco, Chile, was evaluated and it was established how neoliberal urbanism, specifically the touristification of the territory, affects this dimension. Surveys were applied in three units of analysis, resulting in two factors of community tsunami resilience through principal component analysis: "knowledge of the place, networks and tsunami preparedness", and "cohesion and perception of the threat". In addition, social vulnerability, years of residence, morphology and urban typology, elements that influence resilience and are contextualized by the neoliberal management of the territory, were also investigated. U1 resulted in a tourist zone and reached the lowest resilience value; U2 is a tourist-residential zone and obtained an intermediate resilience figure; U3 is a residential zone and reached a higher resilience level. It is established that in those areas with more tourism, social relations are more atomized and there is less relationship with the immediate environment, thus decreasing community resilience to tsunami. It is concluded that, although it is important to emphasize the active role of communities in risk reduction, the causes of disasters respond to structural factors that are beyond the scope of community action, so that actions should be, first and foremost, aimed at reducing vulnerability and exposure.
\end{abstract}

Key words: risk management, tsunami, community resilience, territorial touristification, neoliberal urbanism.

\section{INTRODUCCIÓN}

El riesgo de desastre socio-natural es la probabilidad que en un territorio ocurra un peligro de origen natural denominado amenaza, el cual puede generar daños y pérdidas asociadas a la exposición de comunidades e infraestructura (Romero, 2014), además de niveles específicos de fragilidad que sufren los grupos humanos, diferenciados, a nivel social y territorial, en función de factores políticos, socioeconómicos, institucionales, psicológicos y culturales, situación denominada vulnerabilidad (Thomas, 20131.

Los modelos clásicos de gestión del riesgo de desastre han evolucionado desde el entendimiento del desastre como un evento extremo localizado en el espacio y en el tiempo, hacia el reconocimiento de la vulnerabilidad y la resiliencia como los factores que explican las causas de los desastres, así como su prevención y mitigación (Bacigalupe et al., 2020). Es en este contexto que surge la noción de resiliencia comunitaria, la cual se enfoca en las fortalezas y capacidades inherentes de la comunidad para enfrentar amenazas que les afectan simultáneamente (Flores y Sanhueza, 20181.

Dentro de los enfoques de resiliencia comunitaria se destaca el concepto de capital social (Li Z, 2019; Partelow, 2020), el cual posee un carácter potencial y designa la posibilidad que tienen los individuos de acceder a recursos - sociales, información, psicológicos, financieros y materiales - gracias a las características de los vínculos sociales, lo que se contextualiza por la presencia de grupos formales e informales (Chan et al., 2019), además de confianza, cohesión, percepción de 
inclusión y acción colectiva que permiten vincular las prácticas locales entre los miembros de la comunidad Mallace y Wallace, 2008; Flores y Sanhueza, 2018). A su vez, diversas características y condiciones pueden influir en el capital social, entre ellos, la cantidad de años de residencia en la zona (Livingstone et al., 20081, aspectos del tejido urbano que favorecen la comunicación e interacción entre vecinos (Gonzalez-Muzzio, 2013), así como también el apego al lugar (Sandoval, 2020).

Aún más, existen otros factores que se consideran como factor de resiliencia comunitaria ante el riesgo de desastre, entre ellos, el conocimiento del medio natural y construido del entorno (Gonzalez-Muzzio, 20131, además de la percepción del riesgo, lo cual vincula a la comunidad con el conocimiento de la amenaza y con mayores estrategias de preparación (Navarro et al., 2017).

Se reconoce que, si bien muchas amenazas no son controlables, el fomento de la resiliencia comunitaria puede reducir la vulnerabilidad, disminuyendo consecuentemente el riesgo de desastre (McEntire, 2014). Lo anterior se condice con los señalado por la Directiva Europea de Inundaciones de 2007, la cual recomienda el desarrollo de metodologías e instrumentos para reducir la vulnerabilidad de las comunidades expuestas a peligros naturales (González et al., 2020). Sin embargo, no se debe volver a naturalizar el riesgo mediante enfoques simplistas que obvian las relaciones de poder, despolitizan los mecanismos que construyen la vulnerabilidad, traspasan la responsabilidad de la gestión del riesgo desde el Estado a la misma comunidad y suponen la mera aceptabilidad del riesgo (Fainstein, 2015).

\section{PROBLEMÁTICA}

Debido a las características geológicas, geográficas y climáticas, Chile es un país susceptible a diversas amenazas naturales. Entre ellas, destaca la amenaza de tsunami, la que se contextualiza debido a la localización de la costa frente a una zona de subducción cortical, proceso que involucra la generación de terremotos y tsunamis (Lagos, 2000).

El riesgo de desastre por tsunami toma relevancia debido al aumento de la vulnerabilidad social y la exposición en la zona costera, lo que se debe, entre otras causas, a una planificación territorial omisa a las amenazas naturales, el aumento sostenido de la pobreza y desigualdad social, además de la degradación ambiental (Sandoval, 2020). Lo anterior se da bajo un contexto de Estado neoliberal instaurado en dictadura desde la década de 1980, el cual se ha desplegado través de políticas de desregulación, privatización y mercantilización de los derechos sociales y la gestión territorial (De Mattos, 2013; Carraro et al., 20211.

En las áreas costeras, esta gestión neoliberal del territorio o urbanismo neoliberal ha generado una serie de cambios propiciados por el desarrollo turístico e inmobiliario definido por agentes privados (Guerrero y Alarcón, 2018 ). Bajo esta lógica, la principal transformación es la turistización del espacio (Babinger, 20121, es decir, cambios en el aspecto físico y social del territorio para orientarlo al uso y ocio de los visitantes. Aún más, se ha propiciado el aumento de la exposición a tsunami a través de la construcción en zonas de inundación (Martínez et al., 2020). 
En este escenario de urbanismo neoliberal y de crecimiento exponencial del riesgo ante tsunami, se hace necesario el desarrollo de lineamientos que aborden esta problemática ligada al desastre y, en esta línea, el paradigma de la resiliencia comunitaria ha tomado preponderancia para su generación. Así, el objetivo principal de este artículo es evaluar la resiliencia comunitaria ante tsunami en la comuna de El Quisco, Chile, y establecer cómo el urbanismo neoliberal, específicamente la turistización del territorio, afecta estas dinámicas.

Al respecto, existen luces de cómo la gestión neoliberal del territorio y de los derechos sociales podría estar influyendo en los niveles de resiliencia comunitaria ante tsunami, específicamente por su injerencia en la tipología urbana (Carraro et al., 20211), los años de residencia en la zona (Hidalgo et al., 2016a), la morfología o tejido urbano (Señoret y Link, 20191, además de su intervención en factores claves de la vulnerabilidad social ante el riesgo de desastre, entre ellos educación y vivienda (Martínez et al., 2020), debido al despliegue de lógicas de privatización y competencia que van generando exclusión social (Morales, 2017) y, consecuentemente, menores capacidades para enfrentar los impactos de la amenaza. En torno a este último punto, Chaskin (2016) reconoce relaciones entre el grado de vulnerabilidad social y los niveles de resiliencia comunitaria.

En Chile existen diversos estudios de resiliencia comunitaria ante tsunami IGonzález Muzzio, 2013; Flores y Sanhueza, 2018; Torres et al., 2018; Engel y Warner, 20191, en las cuales se describen y analizan factores inherentes organizacionales y de cooperación dentro del territorio para enfrentar y adaptarse a las consecuencias de esta amenaza. Sin embargo, en estos estudios no se ahonda en las causas socioeconómicas y políticas que configuran el desastre y que obligan a la comunidad a la necesidad de "adaptarse" (Keck y Sakarolrak, 2013). Tampoco se hace alusión a la influencia de aspectos como el turismo masivo en estas dinámicas sociales (Carraro et al., 2021).

Sandoval (2020) propone una (re)apropiación del concepto resiliencia comunitaria, en donde no solo debe entenderse como adaptación y eventual respuesta colectiva frente al desastre por parte de la comunidad, sino como práctica de resistencia y transformación ante los patrones de vulnerabilidad e injusticia territorial generados por el modelo de desarrollo, situación representada en este caso por la exposición a las amenazas naturales y la propia vulnerabilidad o vulnerabilización social.

Siguiendo a Sandoval (2020), en este artículo se identifica la gestión neoliberal del territorio y los derechos sociales como un condicionante del riesgo de tsunami, lo cual lleva a la comunidad a la necesidad de hacer uso de las "características resilientes" en caso de desastre. Reconociendo lo anterior, se pueden generar lineamientos directos e integrales para abordar la reducción del riesgo, tanto a nivel comunitario-local, como a nivel de estructuras políticas y socioeconómicas, principales gatillantes de los desastres desde el enfoque de la ecología política (Carraro et al., 2021).

Así, a través de esta investigación se pretende dar respuesta a las siguientes interrogantes: ¿la turistización del territorio afecta los niveles de resiliencia comunitaria ante tsunami? ¿es suficiente el fortalecimiento de las capacidades 
comunitarias para la reducción del riesgo de tsunami?

En relación a la estructura del artículo, la primera sección abordará la descripción del área de estudio y las implicancias del urbanismo neoliberal en la zona, así como también presentar la metodología utilizada para, por un lado, evaluar la resiliencia comunitaria ante tsunami y por otro, caracterizar la turistización del espacio en la zona estudiada. En el segundo apartado se exponen los principales resultados obtenidos, los cuales son discutidos a través de bibliografía atingente al tema. Finalmente, se exhiben las principales conclusiones del estudio.

\section{MATERIALES Y MÉTODOS ÁREA DE ESTUDIO:}

El Quisco es una comuna-balnerario localizada en la provincia de San Antonio, región de Valparaíso, Chile (Figura $N^{\circ} 1$ ). De acuerdo con el Censo 2017 habitan
15.955 personas, lo que representa un crecimiento demográfico de un $68 \%$ en comparación al Censo 2002.

En torno al proceso de urbanismo neoliberal, este notable crecimiento demográfico se ha visto motivado por la estimulación y venta del imaginario del disfrute de la naturaleza inmerso en condiciones de vida urbana que ofrece la costa -utopolis-, la cual actúa como representaciones que idealizan determinadas relaciones sociales y espaciales (Hidalgo et al., 2016a). En el caso de El Quisco, además, se promueve la idea turístico-residencial del "litoral de los poetas" y destaca la cercanía a Santiago, lo cual profundiza este proceso que se ha materializado, principalmente, en la producción masificada de segundas residencias -viviendas cuyo valor de uso está orientado al ocio y la recreación por más de 24 horas-, además de la aparición de hostales, hoteles y resorts (Hidalgo et al., 2016b).

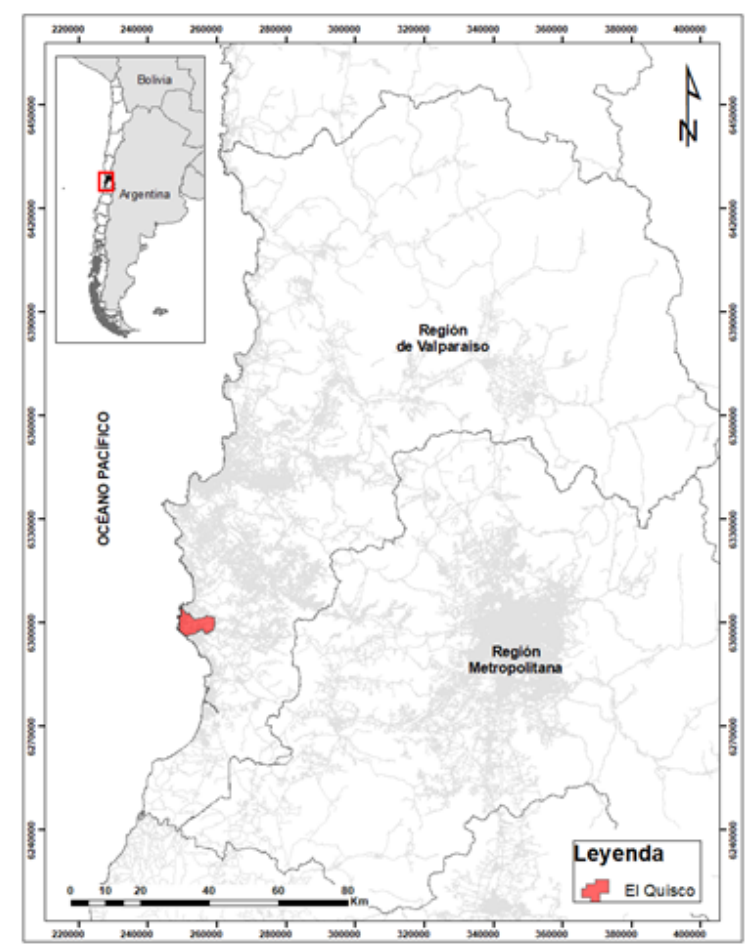

Figura 1. Área de estudio. Fuente: Elaboración propia 
En la comuna ha existido una producción masificada de segundas residencias en el periodo 1992-2002 (Hidalgo et al., $2016 a)$ y una nueva vocación residencial desde el año 2002 orientada a la primarización de segundas residencias y la construcción de viviendas principales (Hidalgo et al., 2016b), lo cual ha establecido nuevas pautas de ocupación del territorio y el aumento del riesgo de desastre ante tsunami. Bajo los 30 m.s.n.m, que según la Oficina Nacional de Emergencia (ONEMI) es la zona que debe evacuar ante un evento extremo de tsunami. El Plan Regulador Comunal permite la construcción de viviendas y equipamiento vecinal, lo cual va configurando un aumento constante de exposición a la amenaza.

La reducción del riesgo de desastre ante tsunami es un desafío para la comuna si se considera un evento similar al terremoto del 8 de julio de 1730, el cual ha sido reconocido como el peor escenario conocido para terremoto y tsunami en la zona central de Chile, con magnitudes en torno a 9.1-9.3 Mw (Carvajal et al., 2017, p.3 en Martínez et al., 2020). De acuerdo con la Carta de Inundación por tsunami (CITSU), elaborada para la comuna de Algarrobo en un escenario similar a 1730 (Servicio Hidrográfico y Oceanográfico de la Armada de Chile y la calle Rosa María.
[SHOA], 2018), parte de la comuna de El Quisco alcanzaría alturas de ola superiores a 6 metros.

\section{METODOLOGÍA:}

El estudio se enfocó en áreas bajo los 30 $m$ s.n.m de parte del sector del centro urbano de El Quisco. A diferencia de otros lugares de la comuna, la geomorfología de terrazas litorales escalonadas configura, en esta zona, mayor superficie expuesta a tsunami, lo cual establece a esta extensión como un espacio con mayor potencial de inundación en todo el municipio (CIREN, 2020).

Se seleccionaron tres unidades de análisis, que corresponden a un conjunto de manzanas censales que comparten ciertas características, principalmente la morfología y tipología urbana. Todas tuvieron como límite la avenida Isidoro Dubornais, al este, y la línea de costa, hacia el oeste (Figura $N^{\circ} 2$ ). La unidad de análisis 1 (en adelante U1) se localizó entre la calle Costanera Sur y Avenida Francia; la unidad de análisis 2 (en adelante U2) se ubicó entre Avenida Francia y La Marina; la unidad de análisis 3 (en adelante U3) se situó entre La Marina 


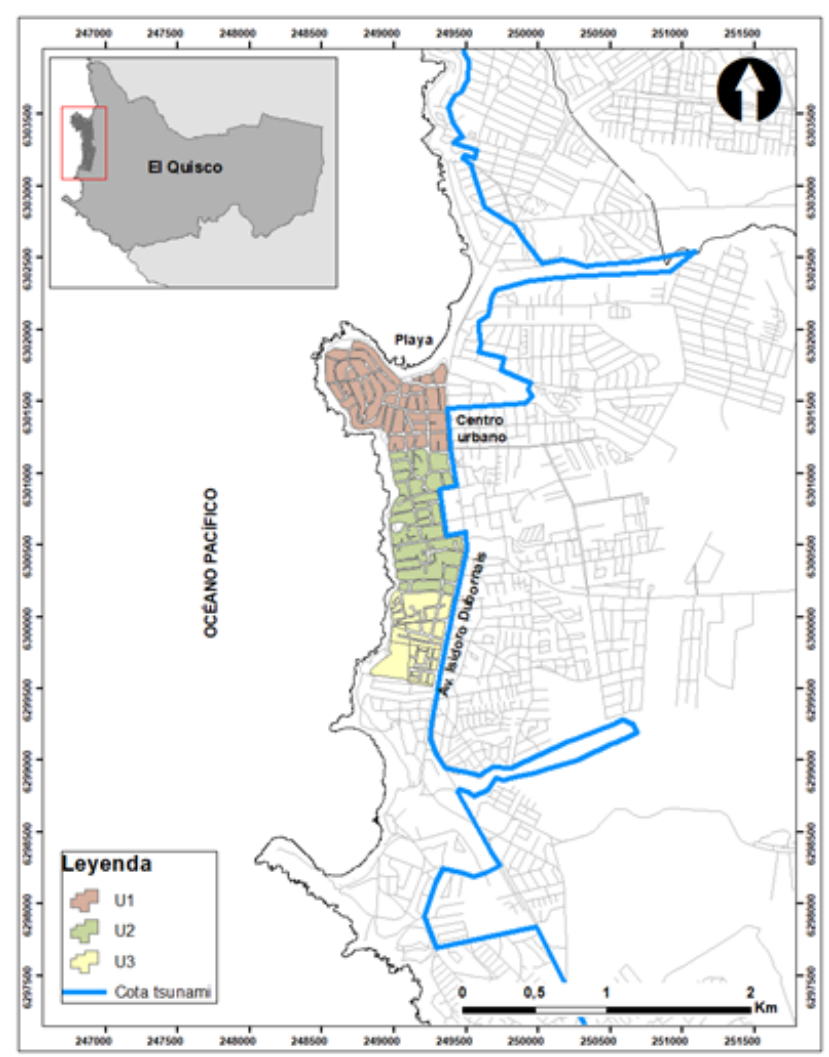

Figura 2. Unidades de análisis. Fuente: Elaboración propia

EVALUACIÓN DE LA RESILIENCIA COMUNITARIA ANTE TSUNAMI

Para la evaluación de la resiliencia comunitaria ante tsunami se aplicaron encuestas de carácter estructurado y se realizó una pregunta específica para cada una de las 10 variables seleccionadas, para lo cual se tomó en consideración dimensiones de capital social, percepción y preparación ante tsunami, además de conocimiento del lugar (Tabla $\mathrm{N}^{0} 1$ ). A cada respuesta se le asignó un puntaje entre 1 y 4 , donde 1 representa el nivel mínimo de evaluación y 4 es el máximo valor por variable. Las encuestas se realizaron a personas mayores de 18 años pertenecientes al hogar. 


\begin{tabular}{|l|l|l|}
\hline \multicolumn{1}{|c|}{ Variable } & \multicolumn{1}{|c|}{ Justificación } & Referencia \\
\hline $\begin{array}{l}\text { Conocimiento de } \\
\text { organizaciones }\end{array}$ & $\begin{array}{l}\text { El conocimiento de } \\
\text { organizaciones se estructura } \\
\text { como potenciales redes de } \\
\text { apoyo, cooperación y nuevos } \\
\text { nodos de organización }\end{array}$ & Chan et al., 2018 \\
\hline Asociatividad & $\begin{array}{l}\text { La particupación voluntaria y } \\
\text { no remunerada al interior de } \\
\text { las redes y proyectos } \\
\text { comunitarios, establece el } \\
\text { grado de autoorganización, } \\
\text { acción colectiva y búsqueda } \\
\text { del bien común }\end{array}$ & Flores y Sanhueza (2018) \\
\hline Trabajo colectivo & $\begin{array}{l}\text { Establece que la comunidad } \\
\text { se trabaja por metas y objetivos } \\
\text { comunes, dando indicios de la } \\
\text { movilización de recursos en la } \\
\text { emergencia }\end{array}$ & Flores y Sanhueza (2018) \\
\hline Confianza & $\begin{array}{l}\text { La confianza promueve los } \\
\text { intercambios voluntarios de } \\
\text { bienes y servicios entre los } \\
\text { actores, ampliando las redes } \\
\text { sociales en la comunidad }\end{array}$ & Flores y Sanhueza (2018) \\
\hline
\end{tabular}

Tabla 1. Variables de resiliencia comunitaria ante tsunami Fuente: Elaboración propia 
El tamaño muestral se estableció de acuerdo análisis. El total de viviendas por manzana con el número de viviendas ocupadas fue de 415, por lo cual se consideró la permanentemente (primeras viviendas) en las siguiente ecuación con un error estándar manzanas censales de las tres unidades de menor al 3\% y $90 \%$ de confiabilidad.

$$
\begin{aligned}
& \text { 1. } n^{\prime}=\frac{s^{2}}{v^{2}} \\
& \text { 2. } n^{\prime}=\frac{n^{\prime}}{7+n^{\prime} / N}
\end{aligned}
$$

Dónde:

n: Tamaño de la muestra

N: Población (viviendas ocupadas permanentemente)

se: Error estándar

s2: Varianza de la muestra

V2: Varianza de la población

p: \% de confiabilidad
En relación con ecuación 1

s2 $=p(1-p)=0,9(1-0,9)=0,09$

$\mathrm{V} 2=(\mathrm{se}) 2=(0,03) 2=0,0009$

Reemplazando los valores en ecuación 1

$n^{\prime}:$ s2 / V2

$n^{\prime}: 0,09 / 0,0009=100$

Luego en ecuación 2 :

$n=n^{\prime} /\left(1+n^{\prime} / N\right)$

$\mathrm{n}=100 /(1+100 / 415)$

$\mathrm{n}=90,4 \sim 90$

Para conocer el número de encuestas por unidad de análisis, se utilizó la siguiente fórmula:

$$
f / h=n / N * N h
$$

Dónde:

$\mathrm{f} / \mathrm{h}=$ cuociente entre $\mathrm{n} / \mathrm{N}$

Nh = número de hogares por manzana en cada unidad de análisis

\begin{tabular}{|c|c|c|}
\hline $\begin{array}{c}\text { Unidad de } \\
\text { Análisis }\end{array}$ & $\begin{array}{c}\text { No de viviendas } \\
\text { ocupadas }\end{array}$ & Encuestas \\
\hline U1 & 90 & 19 \\
\hline U2 & 123 & 27 \\
\hline U3 & 202 & 44 \\
\hline Total & 415 & 90 \\
\hline
\end{tabular}

Tabla 2. Encuestas por unidad de análisis.

Fuente: Elaboración propia 
A partir del número total de encuestas, por unidad de análisis, se generó un promedio por variable. Posteriormente, las variables fueron sometidas a un análisis de componentes principales (ACP) a través del programa estadístico SPSS. Una vez agrupadas en los respectivos componentes resultantes, estos fueron igualmente ponderados para obtener el valor final de resiliencia comunitaria por unidad. Así, los niveles de resiliencia se evaluaron a través del siguiente criterio (Tabla $N^{\circ} 3$ ).

\begin{tabular}{|c|c|}
\hline Valor de resiliencia & Evaluación \\
\hline $1,0-1,59$ & Nula \\
\hline $1,6-2,19$ & Baja \\
\hline $2,2-2,79$ & Media \\
\hline $2,8-3,39$ & Alta \\
\hline$>3,4$ & Muy Alta \\
\hline
\end{tabular}

Tabla 3. Niveles de resiliencia

Fuente: Elaboración propia

Finalmente, se utilizó el software ArcGIS 10.7 para la elaboración de una cartografía que zonifica la resiliencia comunitaria en las tres unidades de análisis.

\section{CARACTERIZACIÓN DE LAS UNIDADES DE ANÁLISIS}

Para establecer cómo el urbanismo neoliberal influye en los niveles de resiliencia comunitaria ante tsunami, las unidades de análisis fueron caracterizadas a partir de diversos parámetros. En primer lugar, a través de una visita a terreno, se fotografiaron hitos característicos de las respectivas unidades, entre ellos, el tipo de vivienda, equipamientos vecinales y los servicios presentes, lo cual permitió establecer la tipología urbana predominante y comprobar si la "turistización del territorio" está presente. Por otro lado, se procedió a indagar el tejido o morfología urbana característica en cada unidad mediante la técnica de fotointerpretación a través de un zoom en la unidad respectiva. Para lo anterior, se hizo uso de imágenes satelitales extraídas de google earth, específicamente una imagen maxar technologies del 15 de septiembre del 2017.

Asimismo, la aplicación de encuestas permitió estimar otras variables asociadas a la gestión neoliberal del territorio y los derechos sociales, las cuales influyen en los niveles de resiliencia, específicamente el nivel educativo como indicador de vulnerabilidad social, además de los años de residencia en la zona.

\section{RESULTADOS}

A nivel general, de las personas encuestadas en las tres unidades de análisis, el 54\% de las es de género femenino y un $46 \%$ pertenece al género masculino (Figura $\mathrm{N}^{\circ} 3$ ). De igual manera, en relación al tramo de edad, 33\% de quienes respondieron tienen más de 60 años, 36\% posee entre 45-59 años, $22 \%$ pertenece al tramo $45-59$ años y sólo 9\% se encuentra entre los 18-29 años. 

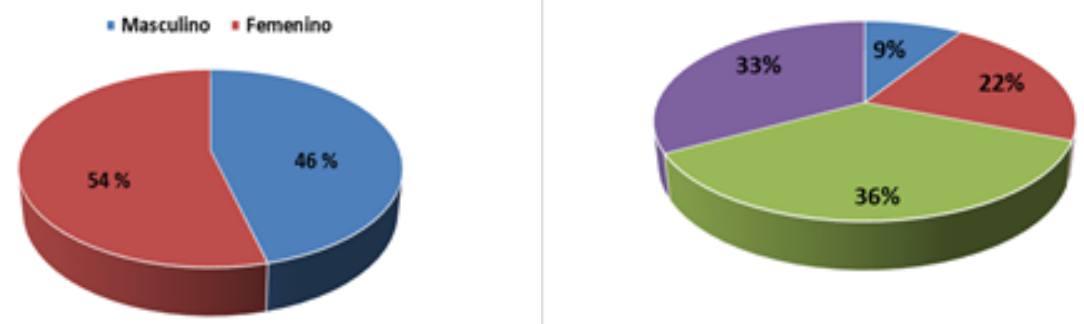

Figura 3. Caracterización de los encuestados por género y edad Fuente: Elaboración propia

NIVELES DE RESILIENCIA

\section{COMUNITARIA ANTE TSUNAMI} POR UNIDAD DE ANÁLISIS:

Desde el análisis de componentes principales aplicado a las 10 variables evaluadas en la encuesta, resultaron dos componentes que explican el 100\% de la varianza. Ambos componentes son interpretados como factores y son los cuales integran la resiliencia comunitaria ante tsunami en la zona. Valores cercanos a 1 o - 1 establecen la pertenencia de la variable al componente (Tabla $\mathrm{N}^{\circ} 4$ ).

\begin{tabular}{|c|c|c|}
\hline \multirow[b]{2}{*}{ Variable } & \multicolumn{2}{|c|}{ Componente } \\
\hline & 1 & 2 \\
\hline Conocimiento de organizaciones &, 995 &, 102 \\
\hline Preparación ante tsunami &, 992 &, 125 \\
\hline $\begin{array}{l}\text { Conocimiento de } \\
\text { recursos físicos del lugar }\end{array}$ &, 991 &, 133 \\
\hline Inclusión &, 947 &, 322 \\
\hline Asociatividad &, 937 &, 349 \\
\hline $\begin{array}{l}\text { Conocimiento de recursos } \\
\text { naturales del lugar }\end{array}$ &,- 862 &, 507 \\
\hline Apego &, 350 &, 937 \\
\hline Confianza &, 383 &, 924 \\
\hline Percepción de la amenaza &, 425 &,- 905 \\
\hline Trabajo colectivo & .682 & ,731 \\
\hline
\end{tabular}

Tabla 4. Matriz del análisis de componentes principales

Fuente: Elaboración propia 
A) Factor 1 - conocimiento del lugar, redes y preparación ante tsunami: explica el $67 \%$ de la varianza total del sistema y está integrado por las variables "conocimiento de los recursos naturales" $y$ "conocimiento de los recursos físicos" en el caso del conocimiento del lugar, "conocimiento de organizaciones", "inclusión" y "asociatividad" con relación a redes, además de la preparación ante tsunami. La U1 es la zona con menor valor de resiliencia en este factor, resultando en 2,2. Por su parte, U2 alcanzó un valor intermedio de 2,3 , mientras que U3 obtuvo el valor más alto entre las tres unidades, alcanzando 2,7 (Tabla N5), lo que establece que, en esta zona, en comparación a las demás estudiadas, existe mejor conocimiento del lugar, mayor participación en redes y destaca la mayor preparación ante tsunami a través de instancias como los simulacros de evacuación.

B) Factor 2 - cohesión y percepción de la amenaza de tsunami: explica el 33\% de la varianza total del sistema y está integrado por las variables "apego", "confianza" y "trabajo colectivo" en el caso de cohesión, además de percepción de la amenaza. UI adquirió la cifra más baja de resiliencia para este factor entre las tres unidades de análisis, logrando 2,7. En relación con U2 alcanzó una cifra de 3,1 , en tanto, U3 obtuvo un valor de 3,25 (Tabla No5), por lo que en esta zona y en predominio a las otras, el nivel de apego e interés por el entorno, la confianza en los vecinos y el trabajo colectivo permite una comunidad más cohesionada, además de presentarse una mayor conciencia que se encuentran habitando en una zona con amenaza latente de tsunami.

C Resiliencia total: U1 adquirió un valor de resiliencia comunitaria ante tsunami de 2,5, por lo que fue evaluada y zonificada como zona con resiliencia media. Por su parte, U2 obtuvo una cifra de 2,7, siendo evaluada, de igual manera, como zona con resiliencia media. Destaca U3, que logró un valor de 3, siendo zonificada como zona con resiliencia alta.

\begin{tabular}{|c|c|c|c|c|}
\hline Unidad & Factor 1 & Factor 2 & Resiliencia total & $\begin{array}{c}\text { Nivel de } \\
\text { resiliencia }\end{array}$ \\
\hline U1 & 2,2 & 2,7 & 2,5 & Media \\
\hline U2 & 2,3 & 3,1 & 2,7 & Media \\
\hline$U 3$ & 2,7 & 3,25 & 3 & Alta \\
\hline
\end{tabular}

Tabla 5. Niveles de resiliencia por unidad de análisis Fuente: Elaboración propia 
Los niveles de resiliencia fueron zonificados en la siguiente cartografía (Figura $\mathrm{N}^{\circ} 4$ ).

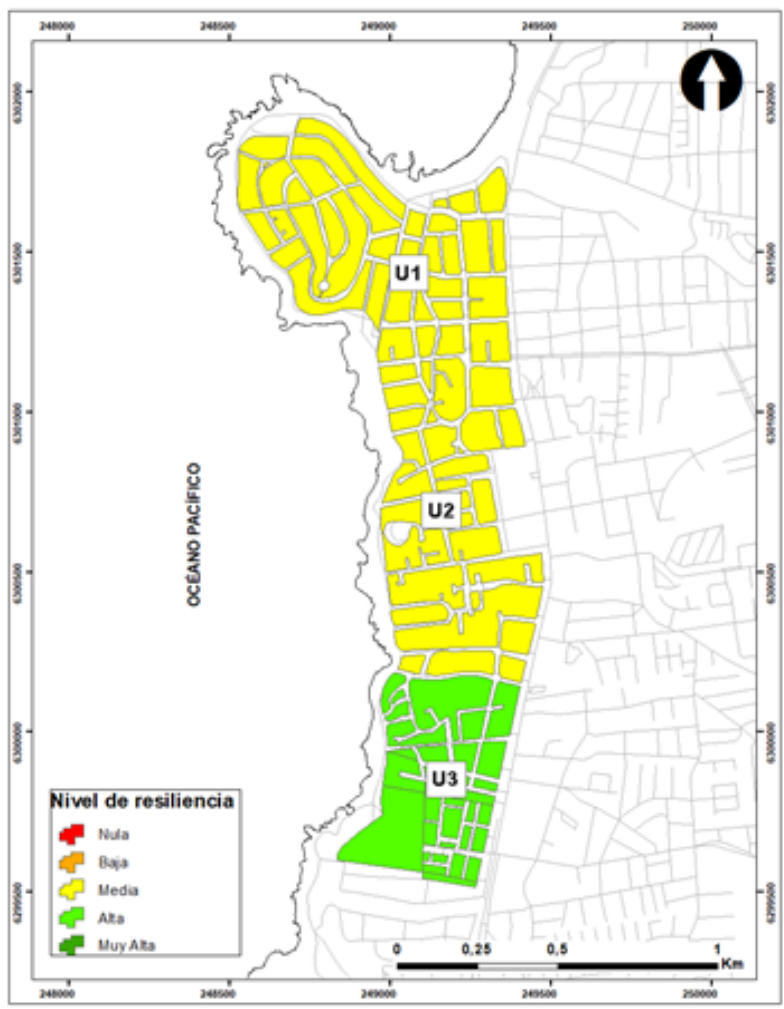

Figura 4. Resiliencia comunitaria por unidad de analisis Fuente: Elaboración propia

\section{CARACTERIZACIÓN DE LAS UNIDADES DE ANÁLISIS}

En relación con la tipología urbana predominante, se estableció que es un área turística con presencia de servicios abocados a esta función, entre ellos paseos peatonales en torno al borde costero, una caleta de pescadores, resorts, hoteles, ferias artesanales, restoranes y pubs (Figura N05.11. Asimismo, existe una gran proporción de segundas residencias, - las que, en época no estival, se caracterizan por poseer todas sus ventanas cerradas y cubiertas con una estructura de madera -. En cuanto a la morfología urbana característica, prevalecen las viviendas dispuestas en manzanas en torno a una red de calles medianas a grandes, referidas, principalmente, al transporte de vehículos motorizados (Figura $N^{\circ} 6.1$ ).

El tiempo de residencia permanente promedio de los habitantes en la zona es de 1 1,5 años, mientras que, en línea al nivel educacional de los encuestados, $68,5 \%$ posee escolaridad completa hasta el nivel de enseñanza media y, además, $21 \%$ cursa o posee nivel educativo técnico superior o profesional.

U2: A partir de la tipología urbana de la unidad, destacó similar proporción de primeras y segundas viviendas, las cuales incluso están agrupadas al interior de condominios y departamentos en la zona más cercana al borde costero. De igual manera, se presentan numerosos servicios 
ligados al turismo, por lo que esta zona fue definida como

transición turístico-residencial (Figura No5.2). En cuanto a la morfología urbana, viviendas y servicios se encuentran dispuestos en manzanas localizadas en torno a una red de calles con ancho mediano a grande, algunas con veredas peatonales (Figura $\mathrm{N}^{\circ}$ 6.21.

El tiempo de residencia permanente en la zona es de 19 años en promedio y, a partir del nivel de escolaridad de los encuestados, $80 \%$ posee escolaridad completa, en donde, además, 48,5\% cuenta con estudios superiores (Tabla $\mathrm{N}^{\circ} 6$ ).

U3: Se estableció como un área netamente residencial, con la característica principal que la mayoría de las viviendas presentes se utilizan de manera permanente y poseen una tipología de vivienda social. Además, se identifican equipamientos típicos de barrio, entre ellos, almacenes y una sede para el funcionamiento de la Junta de Vecinos (Figura $N^{\circ} 5.3$ ). En torno a la morfología urbana, destaca la presencia de pasajes y calles pequeñas, así como también la disposición de espacios públicos, entre ellos, plazas con juegos y bancas (Figura $N^{\circ} 6.3$ ).

El tiempo de residencia permanente en la zona es de 20,5 años y en cuanto a nivel educativo de los encuestados, 50\% logra escolaridad completa y 12,5\% posee 0 está cursando estudios superiores.
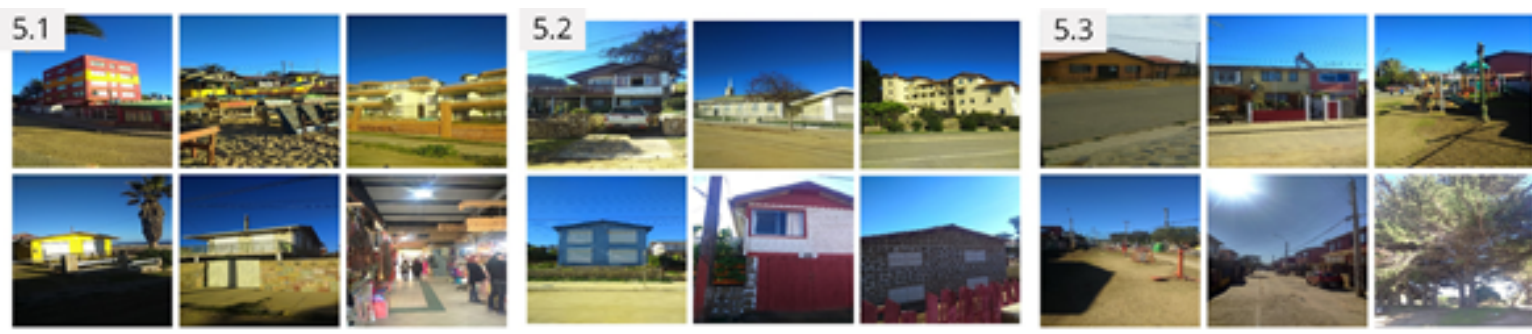

Figura 5. Tipología urbana característica Fuente: Elaboración propia
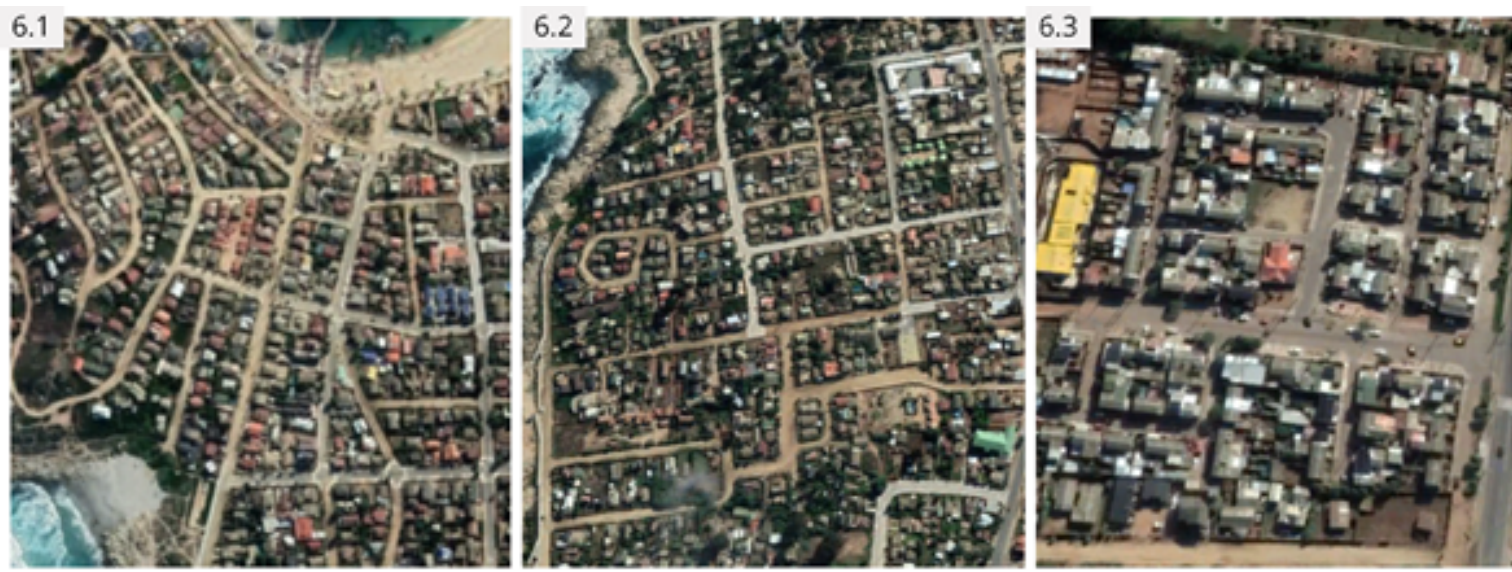

Figura 6. Morfología urbana predominante en cada unidad de análisis Fuente: Elaboración propia 


\begin{tabular}{|c|c|c|}
\hline $\begin{array}{c}\text { Unidad de } \\
\text { análisis }\end{array}$ & $\begin{array}{c}\text { Nivel de escolaridad } \\
\text { completa }\end{array}$ & $\begin{array}{c}\text { Años de residencia } \\
\text { promedio }\end{array}$ \\
\hline U1 & $68,5 \%$ & 11,5 \\
\hline U2 & $80 \%$ & 19 \\
\hline U3 & $50 \%$ & 20,5 \\
\hline
\end{tabular}

Tabla 5. Escolaridad y años de residencia promedio por unidad Fuente: Elaboración propia

\section{DISCUSIÓN}

La diferencia en los niveles de resiliencia comunitaria ante tsunami entre las unidades de análisis es consecuencia de la gestión neoliberal del territorio, fundamentalmente de la turistización del lugar y sus dinámicas (Babinger, 2012) $y$, en esta línea, las áreas en mayor medida "turistizadas" poseen menor nivel de resiliencia. Ul es un área turística o de segunda residencia y alcanzó el valor de resiliencia más bajo; U2 es un área turística-residencial y alcanzó una cifra de resiliencia comunitaria intermedia; U3 es un área netamente residencial o de primera vivienda y obtuvo la cifra y nivel de resiliencia más alta, tanto en los factores evaluados, como a nivel de resiliencia comunitaria total ante tsunami, resultando en una zona con resiliencia alta.

Resiliencia alta significa mejor capacidad asociativa al interior de la comunidad, en donde, además, en mayor medida se valora el conocimiento del territorio, lo que conlleva más posibilidades de acceso a activos, recursos sociales y a normas de comportamiento que podrían garantizar la respuesta y acción colectiva ante el desastre en caso de que se requiera, además de la demanda de soluciones concretas hacia las autoridades para revertir la situación (Romero, 2014). Aún más, es un punto de partida para emprender iniciativas comunitarias durante las distintas fases de la gestión del riesgo de desastre (Navarro et al., 2017), entre ellas y a modo de ejemplo, simulacros de evacuación, identificación de albergues y acopios desde las organizaciones locales, además de charlas educativas y cartografías participativas.

Volviendo a la temática del urbanismo neoliberal (Carraro et al., 2021), existen múltiples

transformaciones socio-territoriales asociadas a la "turistización" del territorio, entre ellas, la configuración de determinadas características en el tejido urbano, las cuales propician el desarrollo de espacios de tránsito para maximizar las dinámicas configuradas por agentes 
privados (Señoret y Link, 2019), en este caso el turismo, a través de vías abocadas a los visitantes para la comunicación directa entre la playa y las segundas viviendas, hostales u hoteles. Lo anterior se comprueba por la adaptación de la trama urbana al transporte de personas y automóviles (Velázquez, 20151, además de la ausencia de espacios públicos y equipamientos urbanos, como juntas de vecinos o clubes deportivos, que propicien la interacción entre la población residente (Hidalgo et al., $2016 a)$.

Al respecto, U3 fue la única en donde fue posible encontrar plazas y equipamientos urbanos tales como Juntas de Vecinos, lo que podría establecer, entre otras variables, el mayor nivel de resiliencia del lugar. Aún más, se destaca que la proximidad física de las viviendas facilitada por morfologías como las encontradas en U3, entre ellas pasajes y calles pequeñas, estimula la comunicación entre vecinos, debido a la mayor probabilidad de interacciones frecuentes (Gonzalez-Muzzio, 2013), consolidando el sistema barrio y dando origen a comunidad (Señoret y Link, 20191.

En tanto en U1 y U2, la morfología urbana predominante corresponde a una red de calles, con ancho variable mediano a grande, en torno a manzanas censales. Este tipo de tejido urbano inhibe la proximidad física, el contacto y la comunicación frecuente entre los habitantes permanentes, lo que, complementando a la ausencia de espacios públicos y la menor proporción de primeras viviendas, dificulta la generación de vínculos entre los vecinos y atomiza las relaciones sociales de este territorio (Señoret y Link, 2019), lo cual se traduce en los menores niveles de resiliencia obtenidos. El binomio ocio-turismo, por el establecimiento de segundas viviendas en este contexto de "turistización", relega a los vínculos de barrio.

Por otro lado, los años de residencia en la zona de igual manera están influidos por el urbanismo neoliberal, lo que se explica, principalmente, por el proceso de primarización de segundas viviendas que comenzó el año 2002 (Hidalgo et al., 2016b). Así, en aquellas áreas "turistizadas" o con mayor presencia de segundas viviendas - espacios en donde este fenómeno se presenta de manera directa - el promedio de años de residencia es menor. En esta línea, según Livingstone et al., (2008) hay menos vínculos e identidad con el entorno inmediato, lo que supone menor apego, cohesión y conocimiento del lugar, tanto de la amenaza de tsunami, así como de los recursos físicos y naturales. Lo anterior se condice con lo identificado en Ul y U3, las cuales son las unidades con menor y mayor años de residencia respectivamente, a la vez que presentan los niveles de resiliencia más bajos y altos entre las áreas estudiadas.

De igual modo, se reconoce que la "turistización" está aumentado sostenidamente la vulnerabilidad social ante el riesgo (Carraro et al., 2021), debido, entre otras causas, a dinámicas de especulación que dificultan el acceso a la vivienda para pobladores locales o de bajos ingresos, aumento del precio de los servicios y el comercio, dinámicas de expulsión y reconversión de la población 
local, además de segregación y exclusión social (Hidalgo et al., 2016b; Guerrero y Alarcón, 20181. Asimismo, se confirma la influencia del neoliberalismo en factores claves de la gestión del riesgo debido a lógicas de exclusión en los derechos sociales (Morales, 2017).

En este estudio, se evaluó el nivel de escolaridad como aproximación a la vulnerabilidad social, ya que menores niveles de educación se traducen en escasas oportunidades y alternativas de desarrollo, además de menores facilidades para entender códigos y mensajes comunicacionales, entre ellos los ligados a la comunicación de riesgos (Labrunée y Gallo, 2005), por lo que es probable que la población con menor escolaridad sea más afectada por los desastres. En esta línea, U3 es el área con menor porcentaje de encuestados con escolaridad completa, 50\% frente a $68,5 \%$ y $80 \%$ de U1 y U2 respectivamente; sin embargo, a su vez, es la unidad que posee mayor nivel de resiliencia comunitaria. Lo anterior, Chaskin (2016) lo reconoce como "efecto barrio", proceso en el cual, en aquellos territorios con mayor vulnerabilidad social, se reconoce la importancia de las relaciones sociales como fuente de solidaridad y apoyo para enfrentar problemas colectivos.

A partir de los resultados obtenidos y la discusión establecida, es importante enfatizar el rol activo de las comunidades en la reducción del riesgo de desastre. Al respecto existen lineamientos para aumentar la resiliencia, las cuales ayudan Los estudios y prácticas de resiliencia al diagnóstico del entorno por parte de la comunitaria deben ser una base para la misma comunidad, además de la co-construcción de conocimiento entre determinación de objetivos y acciones en saberes locales y técnico-científicos, el torno al empoderamiento, la participación y la autodeterminación (Minsoo y Moonhee, 2019). Sin embargo, se debe reconocer que existen límites respecto a lo que pueden lograr las comunidades por sí solas, ya que varios de los factores subyacentes de riesgo son el resultado de procesos estructurales que escapan a las posibilidades del sólo manejo local-territorial (Sandoval, 2020). La resiliencia comunitaria, y los estudios en torno a este enfoque, ocurren, en primer lugar, porque hay población expuesta y vulnerable a las amenazas.

En esta línea, ante todo se debe avanzar en cambios de política concretos, los cuales mitiguen y subsanen los efectos del modelo socioeconómico de desarrollo. Por ejemplo, y en el marco del debate de la nueva Constitución que se presenta en Chile, se debe poner fin al Estado Subsidiario y se tienen garantizar ciertos derechos sociales (Pizarro, 2020), entre ellos, educación, vivienda con perspectiva de gestión del riesgo y salud, lo cual disminuiría la vulnerabilidad-vulnerabilización social al riesgo y permitiría que las personas y comunidades obtengan más herramientas y capacidades para hacerle frente a los desastres. Además, se debe desafiar el marco de gobernanza territorial que impulsa la expansión urbana en área de alto riesgo (Carraro et al., 20211 , por ejemplo, mediante restricciones y la incorporación de áreas de riesgo a los Planes Reguladores Comunales (Romero, 2014). 
desarrollo de métodos participativos e implicativos desde los propios barrios, además de ser un recurso para el análisis de la vulnerabilidad social y un insumo para el desarrollo de una gobernanza de riesgo de desastre sustentada en las formas de vida de los mismos territorios (Escobar, 2007 en Sandoval, 2020).

\section{CONCLUSIONES}

La gestión neoliberal del territorio costero no sólo está influyendo en el aumento de los niveles de exposición y vulnerabilidad social ante desastres, sino que de igual manera está influyendo en las dinámicas socio-territoriales frente al riesgo, evaluado, para objeto de este estudio, a través de la resiliencia comunitaria ante tsunami. La turistización del territorio y sus efectos más inmediatos, como la ausencia de espacios públicos y equipamiento urbano para la interacción entre vecinos, además de la presencia de segundas residencias y el binomio ocio-turismo que ha reemplazado la vida de barrio, consecuentemente ha atomizado las relaciones sociales e influido en la manera de relacionarse con el entorno, repercutiendo en el nivel de conocimiento de lugar, la presencia de organizaciones y participación en redes, además de la preparación ante tsunami, lo que limita la movilización colectiva de recursos en caso de eventual desastre en aquellas áreas más turistizadas.

Si bien es importante relevar el papel activo de las comunidades, el rol central de la reducción del riesgo de desastre no debe caer en las comunidades y, aún más, se debe reconocer que las causas de los desastres responden a factoresestructurales que escapan del control de la comunidad, por lo que las acciones principales deben ir en línea a la reducción de la exposición y la vulnerabilidad. En esta línea, reafirmo el entendimiento de la resiliencia comunitaria como dinámicas colectivas de resistencia y transformación frente a las estructuras de vulnerabilización que configuran el riesgo de desastre, constituyendo un "seguro informal" al que se recurre en caso de necesidad. Así, existe mayor tejido social en los territorios más vulnerables, y, en caso de desastre, es probable que en estos mismos territorios es en donde sea una necesidad activar la movilización colectiva de recursos para hacerle frente. 


\section{REFERENCIAS BIBLIOGRÁFICAS}

Babinger, F. (2012). El turismo ante el reto de peligros naturales recurrentes: una visión desde Cancún. Investigaciones Geográficas, (78), 75-88. http://dx.doi.org/10.14350/rig.32471

Bacigalupe, G., Cienfuegos, R., Cordero, Engel, K., y Warner, J. (2019). Resilience in L., Dammert, L., Ferreiro, A., Inostroza, M., Talcahuano, Chile: appraising local disaster y Tironi, M. (2020). Pensar la pandemia response. Disaster Prevention and Managedesde un nuevo enfoque: 6 propuestas para ment, 2815), 585-602. http://dx. la Gestión del Riesgo de Desastres como doi.org/10.1108/DPM-07-2018-0212 marco de acción para la pandemia y la recuperación. CIGIDEN y Espacio Público. https://espaciopublico.cl/nuestro_trabajo / pensar-la-pandemia-desde-un-nuevo-enfoq ve-b-propuestas-para-la-gestion-del-riesgo-de -desastres-como-marco-de-accion-para-la-pa ndemia-y-la-recuperacion/

Carraro, V., Visconti, C., y Inzunza, S. (2021). Neoliberal urbanism and disaster vulnerability on the Chilean central coast. Geoforum, 121, 89-92. https://doi.org/10.1016/i.geoforum.20 21.02 .023

Centro de Información de Recursos Naturales. (2020). Comuna de El Quisco: recursos naturales. hittps://mmw.sitrural.cl/wp-content/uploads/2020/07/ElQuisco_rec_nat_1.pdf

Chan, NW., Roy, R., Lai, CH., y Tan ML. (2019). Social capital as a vital resource in flood disaster recovery in Malaysia. International Journal of Water Resources Development, 35(4), 619-637. https://doi.org/10.1080/07900627.2018.1467 312

Chaskin, R. (2016). Between the idea and the reality: Public housing reform and the further marginalization of the poor. City \& Community, 15(4), 372-375. hitps://doi.org/10.1111/cico. 12209
De Mattos, C. (2013). Reestructuración económica y metamorfosis urbana en América Latina: de la ciudad a la región urbana. Revis ta del Instituto de la Ciudad, 1(1), 77-100. http://repositorio.flacsoandes.edu.ec/bitstream/10469/6376/1/REXTN-QUR 1-04Mattos.pdf

Fainstein, S. (2015). Resilience and justice. International Journal of urban and regional research, 39111, 157-167. https://doi.org/10.1111/1468-2427.12186

Flores, P., y Sanhueza, R. (2018). Resiliencia comunitaria frente a los desastres naturales: caleta Tumbes, región del Biobío, Chile. Cuadernos de Geografía: Revista Colombiana de Geografía, 27111, 131-145. https://doi.org/10.15446/rcdg.v27n 1.59904

González-Muzzio, C. (2013). El rol del lugar y el capital social en la resiliencia comunitaria posdesastre. Aproximaciones mediante un estudio de caso después del terremoto del 27/F. EURE, 39/117), 25-48. http://dx. doi.org/10.4067/S0250-71612013000 200002

González, L., Baerismyl, S., y Zazo, A. (2020). Resiliencia urbana frente a inundaciones fluviales en Chile: experiencias de San Fernando y Los Ángeles. Revista de urbanismo, (43), 13 1-150. https://dx.doi.org/https://doi.org/10.5354/0717-5051.2020.578 68

Guerrero, R., y Alarcón, M. (2018). Neoliberalismo y transformaciones socio-espaciales en caletas urbanas del Área Metropolitana de Concepción. Los casos de 
aleta Los Bagres y Caleta Cocholgüe, Tomé. Revista de Urbanismo, (38), 1-17. http://dx.doi.org/10.5354/0717-505 1 .2018 .48666

Hidalgo, R., Arenas, F., y Santana, D. (2016a). ¿Utópolis o distópolis?: producción inmobiliaria y metropolización en el litoral central de Chile (1992-2012). EURE, 42(126), 27-54. hitp://dx.doi.org/10.4067/S0250-716120 16000200002

Hidalgo, R., Santana, D., Alvarado, V., Arenas, F., Salazar, A., Valdevenito, C., y Álvarez, L. (2016b). En las costas del Neoliberalismo. Serie Geolibros UC.

Keck, M., y Sakarolrakm, P. (2013). What is social resilience? Lessons learned and ways forward. Erdkunde, 67111, 5-19. http://dx.doi.org/10.3112/erdkunde.20 13.01 .02

Labruneé, M., y Gallo, M. (2013). Vulnerabilidad Social, el camino a la exclusión. Suárez Ediciones.

Lagos, M. (2000). Tsunamis de origen cercano a las costas de Chile. Revista de Geografía Norte Grande, (27), 93-102. https://repositorio.uc.cl/handle/1 1534/ 10436

Li Z, T. (2019). Disaster-recovery social capital and community participation in earthquake-stricken $Y a^{\prime}$ an areas. Sustainability, 11, 1-15. https://doi.org/10.3390/sul 1040993

Livingstone, M., Bailey, N., y Kearns, A. (2008). People's Attachment to Place - The Influence of Neighbourhood Deprivation. Joseph Rowntree Foundation, Chartered Institute of Housing. https://www.jif.org.uk/sites/default/files /iff/migrated/files/2200-neighbourhoodsattachment-deprivation.pdf
Martínez, C., Cienfuegos, R., Inzunza, S., Urrutia, A., y Guerrero, N. (2020). Worst-case tsunami scenario in Cartagena Bay, central Chile: Challenges for coastal risk management. Ocean \& Coastal Management, 185. https://doi.org/10.1016/i.ocecoaman.2019.105060

McEntire, D. (2014). Disaster Response and Recovery: Strategies and Tactics for Resilience $\left(2^{a}\right.$ ed.). University of North Texas.

Minsoo, K., y Moonhee, C. (2019). Examining the role of sense of community: Linking local government public relationships and community-building. Public Relations Review, 45, 297-306. hitps://doi.org/10.1016/j.pubrev.2019.02.002

Morales, J. (2017). Educación y neoliberalismo en Chile: implementación, profundización y crisis (1973-2016). Santiago de Chile: Tesis para optar al grado de Magíster en Filosofía, Facultad de Filosofía y Humanidades, Universidad de Chile.

Navarro, D., Navarro, M., y Vallejo, I. (2017). Resiliencia a desastres y capital social. Análisis de redes sociales en barrios periféricos de la ciudad de Cusco, Perú. Boletín de la Asociación de Geógrafos Españoles, (74),

$10.21138 /$ bage. 2462

Partelow, S. (2020). Social capital and community disaster resilience: postearthquake tourism recovery on Gili Trawangan, Indonesia. Sustain Sci, 16, 203-220. https://doi.org/10.1007/s $11625-020-00$ $854-2$

Pizarro, R. (2020). Chile: rebelión contra el Estado subsidiario. El trimestre económico, 87(346), 333-365. https://. doi.org/10.20430/ete.v87i346.1055 
Romero, H. (2014). Vulnerabilidad, resiliencia y ordenamiento territorial de los desastres socionaturales en Chile. Polígono revista de Geografía, 26(26), 87-1 10. http://dx.doi.org/10.18002/pol.v0i26.1700

Sandoval, J. (2020). Vulnerabilidad-resiliencia ante el proceso de riesgo-desastre: Un análisis desde la ecología política. Polis, 19(56), 138-154. http://dx.doi.org/10.32735/S0718-6568/202 O-N56-1527

Señoret, A. y Link, F. (2019). Densidad urbana, forma y sociabilidad en la ciudad neoliberal: el caso del barrio Santa Isabel en Santiago de Chile. Revista de Urbanismo, 41, 1-17. 10.5354/0717-5051.2019.52862

Servicio Hidrográfico y Oceanográfico de la Armada de Chile. (2018). Carta de Inundación por tsunami, CITSU. Algarrobo $\left(2^{a}\right.$ edición). http://www.shoa.$\mathrm{cl} / \mathrm{s} 3 /$ shoa-cl/descargas/citsu/pdf/ClTSU_Algarrobo_2da_Ed_2018.pdf

Thomas, J. (2013). Evaluación de la vulnerabilidad social ante amenazas naturales en Manzanillo (Colima). Un aporte de método. Investigaciones Geográficas, (81), 79-93.

Torres, M., Cid, B., Bull, M., Moreno, J., Lara, A., González, C., y Henríquez, B. (2018). Resiliencia comunitaria y sentido de comunidad durante la respuesta y recuperación al terremoto-tsunami del año 2010, Talcahuano-Chile. REDER, 2(1), 21-37.

Velázquez, C. (2015). Espacio público y movilidad urbana. Sistemas Integrados de Transporte Masivo (SITM). Tesis doctoral, Programa de Doctorado Espacio Público y Regeneración Urbana. Universidad deBarcelona. https://www.tesisenred.net/bitstream / handle/10803/319707/01.CVVM_1de5 . pdf? sequence $=1$

Wallace, D., y Wallace, R. (2008). Urban Systems during Disasters: Factors for Resilience. Ecology and Society, 13(1). https://www.ecologyandsociety.org/vol 13/iss 1/art 18/ 